\title{
Some nonlinear delay integral inequalities on time scales arising in the theory of dynamics equations
}

\author{
Qinghua Feng ${ }^{12^{*}}$, Fanwei Meng ${ }^{1}$, Yaoming Zhang ${ }^{2}$, Bin Zheng ${ }^{2}$ and Jinchuan Zhou ${ }^{2}$
}

* Correspondence: fqhua@sina.com
'School of Mathematical Sciences,
Qufu Normal University, Qufu,
Shandong, 273165, China
Full list of author information is
available at the end of the article

\begin{abstract}
In this paper, some new nonlinear delay integral inequalities on time scales are established, which provide a handy tool in the research of boundedness of unknown functions in delay dynamic equations on time scales. The established results generalize some of the results in Lipovan [J. Math. Anal. Appl. 322, 349-358 (2006)], Pachpatte [J. Math. Anal. Appl. 251, 736-751 (2000)], Li [Comput. Math. Appl. 59, 1929-1936 (2010)], and Sun [J. Math. Anal. Appl. 301, 265-275 (2005)].
\end{abstract}

MSC 2010: 26E70; 26D15; $26 \mathrm{D} 10$.

Keywords: delay integral inequality, time scales, dynamic equation, bound

\section{Introduction}

In the 1980s, Hilger initiated the concept of time scales [1], which is used as a theory capable to contain both difference and differential calculus in a consistent way. Since then, many authors have expounded on various aspects of the theory of dynamic equations on time scales. For example [2-10], and the references therein. In these investigations, integral inequalities on time scales have been paid much attention by many authors, and a lot of integral inequalities on time scales have been established (see [5-10] and the references therein), which are designed to unify continuous and discrete analysis, and play an important role in the research of boundedness, uniqueness, stability of solutions of dynamic equations on time scales. But to our knowledge, delay integral inequalities on time scales have been paid little attention so far in the literature. Recent results in this direction include the works of $\mathrm{Li}$ [11] and $\mathrm{Ma}$ [12].

Our aim in this paper is to establish some new nonlinear delay integral inequalities on time scales, which are generalizations of some known continuous inequalities and discrete inequalities in the literature. Also, we will present some applications for the established results, in which we will use the present inequalities to derive new bounds for unknown functions in certain delay dynamic equations on time scales.

At first, we will give some preliminaries on time scales and some universal symbols for further use. Throughout this paper, $\mathbf{R}$ denotes the set of real numbers and $\mathbf{R}_{+}=[0, \infty)$, while $\mathbf{Z}$ denotes the set of integers. For two given sets $G, H$, we denote the set of maps from $G$ to $H$ by $(G, H)$.

\section{SpringerOpen ${ }^{\circ}$}

(c) 2011 Feng et al; licensee Springer. This is an Open Access article distributed under the terms of the Creative Commons Attribution License (http://creativecommons.org/licenses/by/2.0), which permits unrestricted use, distribution, and reproduction in any medium, provided the original work is properly cited. 
A time scale is an arbitrary nonempty closed subset of the real numbers. In this paper, $\mathbf{T}$ denotes an arbitrary time scale. On $\mathbf{T}$, we define the forward and backward jump operators $\sigma \in(\mathbf{T}, \mathbf{T})$, and $\rho \in(\mathbf{T}, \mathbf{T})$ such that $\sigma(t)=\inf \{s \in \mathbf{T}, s>t\}, \rho(t)=\sup$ $\{s \in \mathbf{T}, s<t\}$.

Definition 1.1: A point $t \in \mathbf{T}$ is said to be left-dense if $\rho(t)=t$ and $t \neq$ inf $\mathbf{T}$, rightdense if $\sigma(t)=t$ and $t \neq$ sup $\mathbf{T}$, left-scattered if $\rho(t)<t$ and right-scattered if $\sigma(t)>t$.

Definition 1.2: The set $\mathbf{T}^{\kappa}$ is defined to be $\mathbf{T}$ if $\mathbf{T}$ does not have a left-scattered maximum, otherwise it is $\mathbf{T}$ without the left-scattered maximum.

Definition 1.3: A function $f \in(\mathbf{T}, \mathbf{R})$ is called rd-continuous if it is continuous at right-dense points and if the left-sided limits exist at left-dense points, while $f$ is called regressive if $1+\mu(t) f(t) \neq 0$, where $\mu(t)=\sigma(t)-t$. $C_{r d}$ denotes the set of rd-continuous functions, while $\mathcal{R}$ denotes the set of all regressive and rd-continuous functions, and $\mathcal{R}^{+}=\{f \mid f \in \mathcal{R}, 1+\mu(t) f(t)>0, \forall t \in \mathrm{T}\}$.

Definition 1.4: For some $t \in \mathbf{T}^{\kappa}$, and a function $f \in(\mathbf{T}, \mathbf{R})$, the delta derivative of $f$ at $t$ is denoted by $f^{A}(t)$ (provided it exists) with the property such that for every $\varepsilon>0$, there exists a neighborhood $\mathcal{U}$ of $t$ satisfying

$$
\left|f(\sigma(t))-f(s)-f^{\Delta}(t)(\sigma(t)-s)\right| \leq \varepsilon|\sigma(t)-s| \text { for all } s \in \mathcal{U} .
$$

Remark 1.1: If $\mathbf{T}=\mathbf{R}$, then $f^{A}(t)$ becomes the usual derivative $f(t)$, while $f^{A}(t)=f(t+1)$ $f(t)$ if $\mathbf{T}=\mathbf{Z}$, which represents the forward difference.

Definition 1.5: If $F^{\Delta}(t)=f(t), t \in \mathbf{T}^{\kappa}$, then $F$ is called an antiderivative of $f$, and the Cauchy integral of $f$ is defined by

$$
\int_{a}^{b} f(t) \Delta t=F(b)-F(a), \text { where } a, b \in \mathbf{T} .
$$

The following two theorem include some important properties for delta derivative on time scales.

Theorem 1.1 [[13], Theorem 2.2]: If $a, b, c \in \mathbf{T}, \alpha \in \mathbf{R}$, and $f, g \in C_{r d}$, then

(i) $\int_{a}^{b}[f(t)+g(t)] \Delta t=\int_{a}^{b} f(t) \Delta t+\int_{a}^{b} g(t) \Delta t$,

(ii) $\int_{a}^{b}(\alpha f)(t) \Delta t=\alpha \int_{a}^{b} f(t) \Delta t$,

(iii) $\int_{a}^{b} f(t) \Delta t=-\int_{b}^{a} f(t) \Delta t$,

(iv) $\int_{a}^{b} f(t) \Delta t=\int_{a}^{c} f(t) \Delta t+\int_{c}^{b} f(t) \Delta t$,

(v) $\int_{a}^{a} f(t) \Delta t=0$,

(vi) if $f(t) \geq 0$ for all $a \leq t \leq b$, then $\int_{a}^{b} f(t) \Delta t \geq 0$.

For more details about the calculus of time scales, we advise to refer to [14].

\section{Main results}

In the rest of this paper, for the sake of convenience, we denote $\mathbf{T}_{0}=\left[t_{0}, \infty\right) \cap \mathbf{T}$, and always assume $\mathbf{T}_{0} \subset \mathbf{T}^{\kappa}$.

Lemma 2.1 [15]: Assume that $a \geq 0, p \geq q \geq 0$, and $p \neq 0$, then for any $K>0$

$$
a^{\frac{q}{p}} \leq \frac{q}{p} K^{\frac{q-p}{p}} a+\frac{p-q}{p} K^{\frac{q}{p}}
$$


Lemma 2.2: Suppose $u, a \in C_{r d}, m \in \mathcal{R}_{+}, m \geq 0$, and $a$ is nondecreasing. Then,

$$
u(t) \leq a(t)+\int_{t_{0}}^{t} m(s) u(s) \Delta s, \quad t \in \mathbf{T}_{0}
$$

implies

$$
u(t) \leq a(t) e_{m}\left(t, t_{0}\right), \quad t \in \mathbf{T}_{0},
$$

where $e_{m}\left(t, t_{0}\right)$ is the unique solution of the following equation

$$
\gamma^{\Delta}(t)=m(t) y(t), y\left(t_{0}\right)=1 .
$$

Proof: From [[16], Theorem 5.6], we have $u(t) \leq a(t)+\int_{t_{0}}^{t} e_{m}(t, \sigma(s)) a(s) m(s) \Delta s, t \in$ $\mathbf{T}_{0}$. Since $a(t)$ is nondecreasing on $\mathbf{T}_{0}$, then $u(t) \leq a(t)+\int_{t_{0}}^{t} e_{m}(t, \sigma(s)) a(s) m(s) \Delta s \leq a(t)\left[1+\int_{t_{0}}^{t} e_{m}(t, \sigma(s)) m(s) \Delta s\right]$. On the other hand, from [[14], Theorem 2.39 and 2.36 (i)], we have $\int_{t_{0}}^{t} e_{m}(t, \sigma(s)) m(s) \Delta s=\boldsymbol{e}_{m}\left(t, t_{0}\right)-\boldsymbol{e}_{m}(t, t)=\boldsymbol{e}_{m}\left(t, t_{0}\right)-1$. Combining the above information, we can obtain the desired inequality.

Theorem 2.1: Suppose $u, a, b, f \in C_{r d}\left(\mathbf{T}_{0}, \mathbf{R}_{+}\right)$, and $a, b$ are nondecreasing. $\omega \in C\left(\mathbf{R}_{+}, \mathbf{R}_{+}\right)$ is nondecreasing. $\tau \in\left(\mathbf{T}_{0}, \mathbf{T}\right), \tau(t) \leq t,-\infty<\alpha=\inf \left\{\tau(t), t \in \mathbf{T}_{0}\right\} \leq t_{0}, \varphi \in C_{r d}\left(\left[\alpha, t_{0}\right] \cap \mathbf{T}, \mathbf{R}_{+}\right)$. $p>0$ is a constant. If $u(t)$ satisfies, the following integral inequality

$$
u^{p}(t) \leq a(t)+b(t) \int_{t_{0}}^{t} f(s) \omega(u(\tau(s))) \Delta s, \quad t \in \mathbf{T}_{0}
$$

with the initial condition

$$
\left\{\begin{array}{l}
u(t)=\phi(t), t \in\left[\alpha, t_{0}\right] \cap \mathbf{T}, \\
\phi(\tau(t)) \leq a^{\frac{1}{p}}(t), \forall t \in \mathbf{T}_{0}, \quad \tau(t) \leq t_{0},
\end{array}\right.
$$

then

$$
u(t) \leq\left\{G^{-1}\left[G(a(t))+b(t) \int_{t_{0}}^{t} f(s) \Delta s\right]\right\}^{\frac{1}{p}}, \quad t \in \mathbf{T}_{0},
$$

where $G$ is an increasing bijective function, and

$$
G(v)=\int_{1}^{v} \frac{1}{\omega\left(r^{\frac{1}{p}}\right)} d r, v>0 \text { with } G(\infty)=\infty .
$$

Proof: Let $T \in \mathbf{T}_{0}$ be fixed, and

$$
v(t)=a(T)+b(T) \int_{t_{0}}^{t} f(s) \omega(u(\tau(s))) \Delta s .
$$

Then considering $a, b$ are nondecreasing, we have

$$
u(t) \leq v^{\frac{1}{p}}(t), t \in\left[t_{0}, T\right] \cap \mathbf{T} .
$$

Furthermore, for $t \in\left[t_{0}, T\right] \cap \mathbf{T}$, if $\tau(t) \geq t_{0}$, considering $\tau(t) \leq t$, then $\tau_{i}(t) \in\left[t_{0}, T\right]$ $\mathrm{nT}$, and from (6) we obtain

$$
u\left(\tau_{i}(t)\right) \leq v^{\frac{1}{p}}\left(\tau_{i}(t)\right) \leq v^{\frac{1}{p}}(t) .
$$


If $\tau(t) \leq t_{0}$, from (2) we obtain

$$
u(\tau(t))=\phi(\tau(t)) \leq a^{\frac{1}{p}}(t) \leq a^{\frac{1}{p}}(T) \leq v(t) .
$$

So from (7) and (8), we always have

$$
u(\tau(t)) \leq v(t), \quad t \in\left[t_{0}, T\right] \cap \mathbf{T} .
$$

Moreover,

$$
v^{\Delta}(t)=b(T) f(t) \omega(u(\tau(t))) \leq b(T) f(t) \omega\left(v^{\frac{1}{p}}(t)\right),
$$

that is,

$$
\frac{v^{\Delta}(t)}{\omega\left(v^{\frac{1}{p}}(t)\right)} \leq b(T) f(t)
$$

On the other hand, for $t \in\left[t_{0}, T\right] \cap \mathbf{T}$, if $\sigma(t)>t$, then

$$
\begin{aligned}
{[G(v(t))]^{\Delta} } & =\frac{G(v(\sigma(t)))-G(v(t))}{\sigma(t)-t}=\frac{1}{\sigma(t)-t} \int_{v(t)}^{v(\sigma(t))} \frac{1}{\omega\left(r^{\frac{1}{p}}\right)} d r \\
& \leq \frac{v(\sigma(t))-v(t)}{\sigma(t)-t} \frac{1}{\omega\left(v^{\frac{1}{p}}(t)\right)}=\frac{v^{\Delta}(t)}{\omega\left(v^{\frac{1}{p}}(t)\right)} .
\end{aligned}
$$

If $\sigma(t)=t$, then

$$
\begin{aligned}
{[G(v(t))]^{\Delta} } & =\lim _{s \rightarrow t} \frac{G(v(t))-G(v(s))}{t-s}=\lim _{s \rightarrow t} \frac{1}{t-s} \int_{v(s)}^{v(t)} \frac{1}{\omega\left(r^{\frac{1}{p}}\right)} d r \\
& =\lim _{s \rightarrow t} \frac{v(t)-v(s)}{t-s} \frac{1}{\omega\left(\xi^{\frac{1}{p}}\right)}=\frac{v^{\Delta}(t)}{\omega\left(v^{\frac{1}{p}}(t)\right)},
\end{aligned}
$$

where $\xi$ lies between $v(s)$ and $v(t)$. So we always have $[G(v(t))]^{\Delta} \leq \frac{v^{\Delta}(t)}{\omega\left(v^{\frac{1}{p}}(t)\right)}$.

Using the statements above, we deduce that

$$
[G(v(t))]^{\Delta} \leq \frac{v^{\Delta}(t)}{\omega\left(v^{\frac{1}{p}}(t)\right)} \leq b(T) f(t) .
$$

Replacing $t$ with $s$ in the inequality above, and an integration with respect to $s$ from $t_{0}$ to $t$ yields

$$
G(v(t))-G\left(v\left(t_{0}\right)\right) \leq \int_{t_{0}}^{t} b(T) f(s) \Delta s=b(T) \int_{t_{0}}^{t} f(s) \Delta s,
$$

where $G$ is defined in (4).

Considering $G$ is increasing, and $v\left(t_{0}\right)=a(T)$, it follows that

$$
v(t) \leq G^{-1}\left[G(a(T))+b(T) \int_{0}^{t} f(s) \Delta s\right], \quad t \in\left[t_{0}, T\right] \cap \mathbf{T} .
$$


Combining (6) and (12), we get

$$
u(t) \leq\left\{G^{-1}\left[G(a(T))+b(T) \int_{t_{0}}^{t} f(s) \Delta s\right]\right\}^{\frac{1}{p}}, \quad t \in\left[t_{0}, T\right] \cap \mathbf{T} .
$$

Taking $t=T$ in (12), yields

$$
u(T) \leq\left\{G^{-1}\left[G(a(T))+b(T) \int_{t_{0}}^{T} f(s) \Delta s\right]\right\}^{\frac{1}{p}} .
$$

Since $T \in \mathbf{T}_{0}$ is selected arbitrarily, then substituting $T$ with $t$ in (13) yields the desired inequality (3).

Remark 2.1: Since $\mathbf{T}$ is an arbitrary time scale, then if we take $\mathbf{T}$ for some peculiar cases in Theorem 2.1, then we can obtain some corollaries immediately. Especially, if $\mathbf{T}=\mathbf{R}, t_{0}=0$, then Theorem 2.1 reduces to [[17], Theorem 2.2], which is the continuous result. However, if we take $\mathbf{T}=\mathbf{Z}$, we obtain the discrete result, which is given in the following corollary.

Corollary 2.1: Suppose $\mathbf{T}=\mathbf{Z}, n_{0} \in \mathbf{Z}$, and $\mathbf{Z}_{0}=\left[n_{0}, \infty\right) \cap \mathbf{Z} . u, a, b, f \in\left(\mathbf{Z}_{0}, \mathbf{R}_{+}\right)$, and $a, b$ are decreasing on $\mathbf{Z}_{0} . \tau \in\left(\mathbf{Z}_{0}, \mathbf{Z}\right), \tau(n) \leq n,-\infty<\alpha=\inf \left\{\tau(n), n \in \mathbf{Z}_{0}\right\} \leq n_{0}$, $\varphi \in C_{r d}\left(\left[\alpha, n_{0}\right] \cap \mathbf{Z}, \mathbf{R}_{+}\right) . \omega$ is defined the same as in Theorem 2.1. If for $n \in \mathbf{Z}_{0}, u(n)$ satisfies

$$
u^{p}(n) \leq a(n)+b(n) \sum_{s=n_{0}}^{n-1} f(s) \omega(u(\tau(s))), \quad n \in Z_{0},
$$

with the initial condition

$$
\left\{\begin{array}{l}
u(n)=\phi(n), n \in\left[\alpha, n_{0}\right] \cap \mathbf{Z}, \\
\phi(\tau(n)) \leq a^{\frac{1}{p}}(n), \quad \forall n \in \mathbf{Z}_{0}, \quad \tau(n) \leq n_{0},
\end{array}\right.
$$

then

$$
u(n) \leq\left\{G^{-1}\left[G(a(n))+b(n) \sum_{s=n_{0}}^{n-1} f(s)\right]\right\}^{\frac{1}{p}}, \quad n \in \mathbf{Z}_{0} .
$$

In Theorem 2.1, if we change the conditions for $a, b, \omega \mathrm{p}$; then, we can obtain another bound for the function $u(t)$.

Theorem 2.2: Suppose $u, a, b, f \in C_{r d}\left(\mathbf{T}_{0}, \mathbf{R}_{+}\right), \omega \in C\left(\mathbf{R}_{+}, \mathbf{R}_{+}\right)$is nondecreasing, subadditive, and submultiplicative, that is, for $\forall \alpha \geq 0, \beta \geq 0$ we always have $\omega(\alpha+\beta) \leq$ $\omega(\alpha)+\omega(\beta)$ and $\omega(\alpha \beta) \leq \omega(\alpha) \omega(\beta) . \tau, \alpha, \varphi$ are the same as in Theorem 2.1. If $u(t)$ satisfies the inequality (1) with the initial condition (2), then for $\forall K>0$, we have

$$
u(t) \leq\left\{a(t)+b(t) \tilde{G}^{-1}\left[\tilde{G}(A(t))+\int_{t_{0}}^{t} f(s) \omega\left(\frac{1}{p} K^{\frac{1-p}{p}} b(s)\right) \Delta s\right\}^{\frac{1}{p}}, t \in \mathbf{T}_{0},\right.
$$

where $\tilde{G}$ is an increasing bijective function, and

$$
\left\{\begin{array}{l}
\tilde{G}(v)=\int_{1}^{v} \frac{1}{\omega(r)} d r, v>0 \text { with } \tilde{G}(\infty)=\infty, \\
A(t)=\int_{t_{0}}^{t} f(s) \omega\left(\frac{1}{p} K^{\frac{1-p}{p}} a(s)+\frac{p-1}{p} K^{\frac{1}{p}}\right) \Delta s .
\end{array}\right.
$$


Proof: Let

$$
v(t)=\int_{t_{0}}^{t} f(s) \omega(u(\tau(s))) \Delta s, \quad t \in \mathbf{T}_{0}
$$

Then,

$$
u(t) \leq(a(t)+b(t) v(t))^{\frac{1}{P}}, \quad t \in \mathbf{T}_{0} .
$$

Similar to the process of (7)-(9), we have

$$
u(\tau(t)) \leq(a(t)+b(t) v(t))^{\frac{1}{P}}, \quad t \in \mathbf{T}_{0} .
$$

Considering $\omega$ is nondecreasing, subadditive, and submultiplicative, Combining (16), (18), and Lemma 2.1, we obtain

$$
\begin{aligned}
v(t) & \leq \int_{t_{0}}^{t} f(s) \omega\left((a(s)+b(s) v(s))^{\frac{1}{P}}\right) \Delta s \\
& \leq \int_{t_{0}}^{t} f(s) \omega\left(\frac{1}{p} K^{\frac{1-p}{P}}(a(s)+b(s) v(s))+\frac{p-1}{p} K^{\frac{1}{P}}\right) \Delta s \\
& \leq \int_{t_{0}}^{t} f(s) \omega\left(\frac{1}{p} K^{\frac{1-p}{P}} a(s)+\frac{p-1}{p} K^{\frac{1}{P}}\right) \Delta s+\int_{t_{0}}^{t} f(s) \omega\left(\frac{1}{p} K^{\frac{1-p}{P}} b(s)\right) \omega(v(s)) \Delta s \\
& \leq \int_{t_{0}}^{t} f(s) \omega\left(\frac{1}{p} K^{\frac{1-p}{P}} a(s)+\frac{p-1}{p} K^{\frac{1}{P}}\right) \Delta s+\int_{t_{0}}^{t} f(s) \omega\left(\frac{1}{p} K^{\frac{1-p}{P}} b(s)\right) \omega(v(s)) \Delta s \\
& =A(t)+\int_{t_{0}}^{t} f(S) \omega\left(\frac{1}{p} K^{\frac{1-p}{P}} b(s)\right) \omega(v(s)) \Delta s, \forall K>0, t \in \mathbf{T}_{0},
\end{aligned}
$$

where $A(t)$ is defined in (15).

Let $T$ be fixed in $\mathbf{T}_{0}$, and $t \in\left[t_{0}, T\right] \cap \mathbf{T}$. Denote

$$
z(t)=A(T)+\int_{t_{0}}^{t} f(S) \omega\left(\frac{1}{p} K^{\frac{1-p}{P}} b(s)\right) \omega(v(s)) \Delta s,
$$

Considering $A(t)$ is nondecreasing, then we have

$$
v(t) \leq z(t), \quad t \in\left[t_{0}, T\right] \cap \mathbf{T} .
$$

Furthermore,

$$
z^{\Delta}(t)=f(t) \omega\left(\frac{1}{p} K^{\frac{1-p}{P}} b(t)\right) \omega(v(t)) \leq f(t) \omega\left(\frac{1}{p} K^{\frac{1-p}{P}} b(t)\right) \omega(Z(t)) .
$$

Similar to Theorem 2.1, we have

$$
[\tilde{G}(z(t))]^{\Delta} \leq \frac{z^{\Delta}(t)}{\omega(z(t))} \leq f(t) \omega\left(\frac{1}{p} K^{\frac{1-p}{P}} b(t)\right) .
$$

Substituting $t$ with $s$ in (22), and an integration with respect to $s$ from $t_{0}$ to $t$ yields

$$
\tilde{G}(z(t))-\tilde{G}\left(z\left(t_{0}\right)\right) \leq \int_{t_{0}}^{t} f(s) \omega\left(\frac{1}{p} K^{\frac{1-p}{P}} b(s)\right) \Delta s,
$$

which is followed by

$$
\begin{aligned}
z(t) & \leq \tilde{G}^{-1}\left[\tilde{G}\left(z\left(t_{0}\right)\right)+\int_{t_{0}}^{t} f(s) \omega\left(\frac{1}{p} K^{\frac{1-p}{p}} b(S)\right) \Delta s\right] \\
& =\tilde{G}^{-1}\left[\tilde{G}(A(T))+\int_{t_{0}}^{t} f(s) \omega\left(\frac{1}{p} K^{\frac{1-p}{p}} b(S)\right) \Delta s\right] .
\end{aligned}
$$


Combining (17), (21), and (23), we obtain

$$
u(t) \leq\left\{a(t)+b(t) \tilde{G}^{-1}\left[\tilde{G}(A(T))+\int_{t_{0}}^{t} f(s) \omega\left(\frac{1}{p} K^{\frac{1-p}{p}} b(s)\right) \Delta s\right\}^{\frac{1}{p}}, t \in\left[t_{0}, T\right] \bigcap \mathbf{T} .\right.
$$

Taking $t=T$ in (24), yields

$$
u(T) \leq\left\{a(T)+b(T) \tilde{G}^{-1}\left[\tilde{G}(A(T))+\int_{t_{0}}^{T} f(s) \omega\left(\frac{1}{p} K^{\frac{1-p}{p}} b(s)\right) \Delta s\right\}^{\frac{1}{p}} .\right.
$$

Since $T$ is selected from $\mathbf{T}_{0}$ arbitrarily, then substituting $T$ with $t$ in (25), we can obtain the desired inequality (14).

Remark 2.2: Theorem 2.2 unifies some known results in the literature. If we take $\mathbf{T}=\mathbf{R}, t_{0}=0, \tau(t)=t, K=1$, then Theorem 2.2 reduces to [[18], Theorem 2(b3)], which is one case of continuous inequality. If we take $\mathbf{T}=\mathbf{Z}, t_{0}=0, \tau(t)=t, K=1$, then Theorem 2.2 reduces to [[18], Theorem 4(d3)], which is the discrete analysis of [[18], Theorem 2(b3)].

Now we present a more general result than Theorem 2.1. We study the following delay integral inequality on time scales.

$$
\eta(u(t)) \leq a(t)+b(t) \int_{t_{0}}^{t}\left[f(s) \omega\left(u\left(\tau_{1}(s)\right)\right)+g(s) \int_{t_{0}}^{s} h(\xi) \omega\left(u\left(\tau_{2}(\xi)\right)\right) \Delta \xi\right] \Delta s, \quad t \in \mathbf{T}_{0},
$$

where $u, a, b, f, g, h \in C r d\left(\mathbf{T}_{0}, \mathbf{R}_{+}\right), \omega \in C\left(\mathbf{R}_{+}, \mathbf{R}_{+}\right)$, and $a, b, \omega$ are nondecreasing, $\eta \in C\left(\mathbf{R}_{+}, \mathbf{R}_{+}\right)$is increasing, $\tau_{i} \in\left(\mathbf{T}_{0}, \mathbf{T}\right)$ with $\tau_{i}(t) \leq t, i=1,2$, and $-\infty<\alpha=\inf \left\{\min \left\{\tau_{i}\right.\right.$ $\left.(t), i=1,2\}, t \in \mathbf{T}_{0}\right\} \leq t_{0}$.

Theorem 2.3: Define a bijective function $\widehat{G} \in\left(\mathbf{R}_{+}, \mathbf{R}\right)$ such that $\widehat{G}(v)=\int_{1}^{v} \frac{1}{\omega\left(\eta^{-1}(r)\right)} d r, v>0$, with $\widehat{G}(\infty)=\infty$. If $\widehat{G}$ is increasing, and for $t \in \mathbf{T}_{0}, u$ $(t)$ satisfies the inequality (26) with the initial condition

$$
\left\{\begin{array}{l}
\eta(u(t))=\phi(t), \quad t \in\left[\alpha, t_{0}\right] \cap \mathbf{T} \\
\phi\left(\tau_{i}(t)\right) \leq a(t), \quad \forall t \in \mathbf{T}_{0}, \quad \tau_{i}(t) \leq t_{0}, \quad i=1,2
\end{array}\right.
$$

where $\varphi \in C_{r d}\left(\left[\alpha, t_{0}\right] \cap \mathbf{T}, \mathbf{R}_{+}\right)$, then

$$
u(t) \leq \eta^{-1}\left\{\widehat{G}^{-1}\left\{\widehat{G}(a(t))+b(t) \int_{t_{0}}^{t}\left[f(s)+g(s) \int_{t_{0}}^{s} h(\xi) \Delta \xi\right] \Delta s\right\}\right\}, \quad t \in \mathbf{T}_{0} .
$$

Proof: Let the right side of (26) be $v(t)$, then

$$
\eta(u(t)) \leq v(t), \quad t \in \mathbf{T}_{0} .
$$

For $t \in \mathbf{T}_{0}$, if $\tau_{i}(t) \geq t_{0}$, considering $\tau_{i}(t) \leq t$, then $\tau_{i}(t) \in \mathbf{T}_{0}$, and from (29), we have

$$
\eta\left(u\left(\tau_{i}(t)\right)\right) \leq v\left(\tau_{i}(t)\right) \leq v(t) .
$$

If $\tau_{i}(t) \leq t_{0}$, from (27), we obtain

$$
\eta\left(u\left(\tau_{i}(t)\right)\right)=\phi\left(\tau_{i}(t)\right) \leq a(t) \leq v(t) .
$$

So from (30) and (31), we always have

$$
\eta\left(u\left(\tau_{i}(t)\right)\right) \leq v(t), \quad i=1,2 \forall t \in \mathbf{T}_{0} .
$$


Furthermore, considering $\eta$ is increasing, we get that

$$
v(t) \leq a(t)+b(t) \int_{t_{0}}^{t}\left[f(s) \omega\left(\eta^{-1}(v(s))\right)+g(s) \int_{t_{0}}^{s} h(\xi) \omega\left(\eta^{-1}(v(\xi))\right) \Delta \xi\right] \Delta s, \quad t \in \mathbf{T}_{0} .
$$

Fix a $T \in \mathbf{T}_{0}$, and let $t \in\left[t_{0}, T\right] \cap \mathbf{T}$. Define

$$
c(t)=a(T)+b(T) \int_{t_{0}}^{t}\left[f(s) \omega\left(\eta^{-1}(v(s))\right)+g(s) \int_{t_{0}}^{s} h(\xi) \omega\left(\eta^{-1}(v(\xi))\right) \Delta \xi\right] \Delta s,
$$

Since $a, b$ are nondecreasing on $\mathbf{T}_{0}$, it follows that

$$
v(t) \leq c(t), \quad t \in\left[t_{0}, T\right] \cap \mathbf{T} .
$$

On the other hand,

$$
\begin{aligned}
c^{\Delta}(t) & =b(T)\left[f(t) \omega\left(\eta^{-1}(v(t))\right)+g(t) \int_{t_{0}}^{t} h(\xi) \omega\left(\eta^{-1}(v(\xi))\right) \Delta \xi\right] \\
& \leq b(T)\left[f(t) \omega\left(\eta^{-1}(c(t))\right)+g(t) \int_{t_{0}}^{t} h(\xi) \omega\left(\eta^{-1}(c(\xi))\right) \Delta \xi\right] \\
& \leq b(T)\left[f(t)+g(t) \int_{t_{0}}^{t} h(\xi) \Delta \xi\right] \omega\left(\eta^{-1}(c(t))\right) .
\end{aligned}
$$

Similar to Theorem 2.1, we have

$$
[\widehat{G}(c(t))]^{\Delta} \leq \frac{c^{\Delta}(t)}{\omega\left(\eta^{-1}(c(t))\right)} \leq b(T)\left[f(t)+g(t) \int_{t_{0}}^{t} h(\xi) \Delta \xi\right]
$$

Replacing $t$ with $\mathrm{s}$, and an integration for (36) with respect to $s$ from $t_{0}$ to $t$ yields

$$
\widehat{G}(c(t))-\widehat{G}\left(c\left(t_{0}\right)\right) \leq b(T) \int_{t_{0}}^{t}\left[f(s)+g(s) \int_{t_{0}}^{s} h(\xi) \Delta \xi\right] \Delta s .
$$

Since $c\left(t_{0}\right)=a(T)$, and $G$ is increasing, it follows that

$$
c(t) \leq \widehat{G}^{-1}\left\{\widehat{G}(a(T))+b(T) \int_{t_{0}}^{t}\left[f(s)+g(s) \int_{t_{0}}^{s} h(\xi) \Delta \xi\right] \Delta s\right\}
$$

Combining (29), (35), (38), we have

$$
u(t) \leq \eta^{-1}\left\{\widehat{G}^{-1}\left\{\widehat{G}(a(T))+b(T) \int_{t_{0}}^{t}\left[f(s)+g(s) \int_{t_{0}}^{s} h(\xi) \Delta \xi\right] \Delta s\right\}\right\}, \quad t \in\left[t_{0}, T\right] \cap \mathbf{T} .
$$

Taking $t=T$ in (39), yields

$$
u(T) \leq \eta^{-1}\left\{\widehat{G}^{-1}\left\{\widehat{G}(a(T))+b(T) \int_{t_{0}}^{T}\left[f(s)+g(s) \int_{t_{0}}^{s} h(\xi) \Delta \xi\right] \Delta s\right\}\right\} .
$$

Since $T \in \mathbf{T}_{0}$ is selected arbitrarily, then substituting $T$ with $t$ in (40) yields the desired inequality (28).

Remark 2.3: If we take $\eta(u)=u^{p}, g(t) \equiv 0$, then Theorem 2.3 reduces to Theorem 2.1. Next, we consider the delay integral inequality of the following form.

$$
u^{p}(t) \leq a(t)+\int_{t_{0}}^{t}\left[m(s)+f(s) u^{p}\left(\tau_{1}(s)\right)+g(s) \omega\left(u\left(\tau_{2}(s)\right)\right)+\int_{t_{0}}^{s} h(\xi) \omega\left(u\left(\tau_{2}(\xi)\right)\right) \Delta \xi\right] \Delta s,
$$

where $u, f, g, h, a, \tau_{i}, i=1,2$ are the same as in Theorem $2.3, m \in C\left(\mathbf{R}_{+}, \mathbf{R}_{+}\right), p>0$ is a constant, $\omega \in C\left(\mathbf{R}_{+}, \mathbf{R}_{+}\right)$is nondecreasing, and $\omega$ is submultitative, that is, $\omega(\alpha \beta) \leq$ $\omega(\alpha) \omega(\beta)$ holds for $\forall \alpha \geq 0, \beta \geq 0$. 
Theorem 2.4: Suppose $G \in\left(\mathbf{R}_{+}, \mathbf{R}\right)$ is an increasing bijective function defined as in Theorem 2.1. If $u(t)$ satisfies, the inequality (41) with the initial condition

$$
\left\{\begin{array}{l}
u(t)=\phi(t), t \in\left[\alpha, t_{0}\right] \cap \mathbf{T}, \\
\phi\left(\tau_{i}(t)\right) \leq a^{\frac{1}{p}}(t), \quad \forall t \in \mathbf{T}_{0}, \quad \tau_{i}(t) \leq t_{0}, i=1,2,
\end{array}\right.
$$

then

$$
\begin{aligned}
u(t) \leq & \left\{G^{-1}\left\{G\left[a(t)+\int_{t_{0}}^{t} m(s) \Delta s\right]+\int_{t_{0}}^{t}\left[g(s)+\int_{t_{0}}^{s} h(\xi) \Delta \xi\right] \omega\left(e_{f}^{\frac{1}{P}}\left(s, t_{0}\right)\right) \Delta s\right\}\right. \\
& \left.e_{f}\left(t, t_{0}\right)\right\}^{\frac{1}{p}}, \quad t \in \mathbf{T}_{0} .
\end{aligned}
$$

Proof: Let the right side of (41) be $v(t)$. Then,

$$
u(t) \leq v^{\frac{1}{p}}(t), \quad t \in \mathbf{T}_{0}
$$

and similar to the process of (30)-(32) we have

$$
u\left(\tau_{i}(t)\right) \leq v^{\frac{1}{p}}(t), \quad i=1,2 \quad t \in \mathbf{T}_{0} .
$$

Furthermore,

$$
v(t) \leq a(t)+\int_{t_{0}}^{t}\left[m(s)+g(s) \omega\left(v^{\frac{1}{p}}(s)\right)+\int_{t_{0}}^{s} h(\xi) \omega\left(v^{\frac{1}{p}}(\xi)\right) \Delta \xi\right] \Delta s+\int_{t_{0}}^{t} f(s) v(s) \Delta s .
$$

A suitable application of Lemma 2.2 to (46) yields

$$
v(t) \leq\left\{a(t)+\int_{t_{0}}^{t}\left[m(s)+g(s) \omega\left(v^{\frac{1}{p}}(s)\right)+\int_{t_{0}}^{s} h(\xi) \omega\left(v^{\frac{1}{p}}(\xi)\right) \Delta \xi\right] \Delta s\right\} e_{f}\left(t, t_{0}\right) .
$$

Fix a $T \in \mathbf{T}_{0}$, and let $t \in\left[t_{0}, T\right] \cap \mathbf{T}$. Define

$$
c(t)=a(T)+\int_{t_{0}}^{T} m(s) \Delta s+\int_{t_{0}}^{t}\left[g(s) \omega\left(v^{\frac{1}{p}}(s)\right)+\int_{t_{0}}^{t} h(\xi) \omega\left(v^{\frac{1}{p}}(\xi)\right) \Delta \xi\right] \Delta s .
$$

Then,

$$
v(t) \leq c(t) e_{f}\left(t, t_{0}\right), \quad t \in\left[t_{0}, T\right] \cap \mathbf{T},
$$

and

$$
\begin{aligned}
& c^{\Delta}(t)=g(t) \omega\left(v^{\frac{1}{p}}(t)\right)+\int_{t_{0}}^{t} h(\xi) \omega\left(v^{\frac{1}{p}}(\xi)\right) \Delta \xi \leq\left[g(t)+\int_{t_{0}}^{t} h(\xi) \Delta \xi\right] \omega\left(v^{\frac{1}{p}}(t)\right) \\
& \leq\left[g(t)+\int_{t_{0}}^{t} h(\xi) \Delta \xi\right] \omega\left(c^{\frac{1}{p}}(t) e_{f}^{\frac{1}{p}}\left(t, t_{0}\right)\right) \leq\left[g(t)+\int_{t_{0}}^{t} h(\xi) \Delta \xi\right] \omega\left(c^{\frac{1}{p}}(t)\right) \omega\left(e_{f}^{\frac{1}{p}}\left(t, t_{0}\right)\right) .
\end{aligned}
$$

Similar to Theorem 2.1, we have

$$
[G(c(t))]^{\Delta} \leq \frac{c^{\Delta}(t)}{\omega\left(c^{\frac{1}{p}}(t)\right)} \leq\left[g(t)+\int_{t_{0}}^{t} h(\xi) \Delta \xi\right] \omega\left(e_{f}^{\frac{1}{p}}\left(t, t_{0}\right)\right) .
$$


An integration for (50) from $t_{0}$ to $t$ yields

$$
G(c(t))-G\left(c\left(t_{0}\right)\right) \leq \int_{t_{0}}^{t}\left[g(s)+\int_{t_{0}}^{s} h(\xi) \Delta \xi\right] \omega\left(e_{f}^{\frac{1}{p}}\left(s, t_{0}\right)\right) \Delta s,
$$

Considering $G$ is increasing and $c\left(t_{0}\right)=a(T)+\int_{t_{0}}^{T} m(s) \Delta s$, it follows

$$
\begin{aligned}
c(t) \leq & G^{-1}\left\{G\left[a(T)+\int_{t_{0}}^{T} m(s) \Delta s\right]+\int_{t_{0}}^{t}\left[g(s)+\int_{t_{0}}^{s} h(\xi) \Delta \xi\right] \omega\left(e_{f}^{\frac{1}{p}}\left(s, t_{0}\right)\right) \Delta s\right\}, \\
& t \in\left[t_{0}, T\right] \cap \mathbf{T} .
\end{aligned}
$$

Combining (44), (49), and (51), we have

$$
\begin{aligned}
u(t) \leq & \left\{G^{-1}\left\{G\left[a(T)+\int_{t_{0}}^{T} m(s) \Delta s\right]+\int_{t_{0}}^{t}\left[g(s)+\int_{t_{0}}^{s} h(\xi) \Delta \xi\right] \omega\left(e_{f}^{\frac{1}{p}}\left(s, t_{0}\right)\right) \Delta s\right\}\right. \\
& \left.e_{f}\left(t, t_{0}\right)\right\}^{\frac{1}{p}}, t \in\left[t_{0}, T\right] \cap \mathbf{T} .
\end{aligned}
$$

Taking $t=T$ in (52), yields

$$
\begin{aligned}
u(T) \leq & \left\{G^{-1}\left\{G\left[a(T)+\int_{t_{0}}^{T} m(s) \Delta s\right]+\int_{t_{0}}^{T}\left[g(s)+\int_{t_{0}}^{s} h(\xi) \Delta \xi\right] \omega\left(_{f}^{\frac{1}{p}}\left(s, t_{0}\right)\right) \Delta s\right\}\right. \\
& \left.e_{f}\left(T, t_{0}\right)\right\}^{\frac{1}{p}} .
\end{aligned}
$$

Since $T \in \mathbf{T}_{0}$ is selected arbitrarily, after substituting $T$ with $t$ in (53), we obtain the desired inequality (43).

Remark 2.4: If we take $\omega(u)=u, \tau_{1}(t)=t, h(t) \equiv 0$, then Theorem 2.4 reduces to [[11], Theorem 3]. If we take $m(t)=f(t)=h(t) \equiv 0$, then Theorem 2.4 reduces to Theorem 2.1 with slight difference.

Finally, we consider the following integral inequality on time scales.

$$
u^{p}(t) \leq C+\int_{t_{0}}^{t}\left[f(s) u^{q}\left(\tau_{1}(s)\right)+g(s) u^{q}\left(\tau_{2}(s)\right) \omega\left(u\left(\tau_{2}(s)\right)\right)\right] \Delta s, \quad t \in \mathbf{T}_{0},
$$

where $u, f, g, \omega, \tau_{1}, \tau_{2}$ are the same as in Theorem 2.3, $p, q, C$ are constants, and $p>q$ $>0, C>0$.

Theorem 2.5: If $u(t)$ satisfies (54) with the initial condition (42), then

$$
u(t) \leq\left\{\bar{G}^{-1}\left\{H^{-1}\left[H\left(\bar{G}(C)+\int_{t_{0}}^{t} f(s) \Delta s\right)+\int_{t_{0}}^{t} g(s) \Delta s\right]\right\}\right\}^{\frac{1}{p}}, \quad t \in \mathbf{T}_{0},
$$

where $\bar{G}, H$ are two increasing bijective functions, and

$$
\bar{G}(v)=\int_{1}^{v} \frac{1}{r^{\frac{q}{p}}} d r, v>0, H(z)=\int_{1}^{z} \frac{1}{\omega\left(\left(\bar{G}^{-1}(r)\right)^{\frac{1}{p}}\right)} d r, z>0 \text { with } H(\infty)=\infty .
$$

Proof: Let the right side of (54) be $v(t)$. Then,

$$
u(t) \leq v^{\frac{1}{p}}(t), \quad t \in \mathbf{T}_{0}
$$

and similar to the process of (30)-(32) we have

$$
u\left(\tau_{i}(t)\right) \leq v(t), \quad i=1,2 \quad t \in \mathbf{T}_{0} .
$$


Furthermore,

$$
v^{\Delta}(t)=f(t) u^{q}\left(\tau_{1}(t)\right)+g(s) u^{q}\left(\tau_{2}(t)\right) \omega\left(u\left(\tau_{2}(t)\right)\right) \leq f(t) v^{\frac{q}{p}}(t)+g(s) v^{\frac{q}{p}}(t) \omega\left(v^{\frac{1}{p}}(t)\right) .
$$

Similar to Theorem 2.1, we have

$$
[\bar{G}(v(t))]^{\Delta} \leq \frac{v^{\Delta}(t)}{v^{\frac{q}{p}}(t)} \leq f(t)+g(t) \omega\left(v^{\frac{1}{p}}(t)\right) .
$$

An integration for (59) from $t_{0}$ to $t$ yields

$$
\bar{G}(v(t))-\bar{G}\left(v\left(t_{0}\right)\right) \leq \int_{t_{0}}^{t}\left[f(s)+g(s) \omega\left(v^{\frac{1}{p}}(s)\right)\right] \Delta s .
$$

Considering $\bar{G}$ is increasing, and $v\left(t_{0}\right)=C$, then (60) implies

$$
v(t) \leq \bar{G}^{-1}\left[\bar{G}(C)+\int_{t_{0}}^{t}\left[f(s)+g(s) \omega\left(v^{\frac{1}{p}}(s)\right)\right] \Delta s\right] .
$$

Given a fixed number $T$ in $\mathbf{T}_{0}$, and $t \in\left[t_{0}, T\right]$. Let

$$
z(t)=\bar{G}(C)+\int_{t_{0}}^{T} f(s) \Delta s+\int_{t_{0}}^{t} g(s) \omega\left(v^{\frac{1}{p}}(s)\right) \Delta s .
$$

Then,

$$
v(t) \leq \overline{\mathrm{G}}^{-1}(z(t)), t \in\left[t_{0}, \quad T\right] \cap \mathbf{T},
$$

and furthermore,

$$
z^{\Delta}(t)=g(t) \omega\left(v^{\frac{1}{p}}(t)\right) \leq g(t) \omega\left(\left(\bar{G}^{-1}(z(t))\right)^{\frac{1}{p}}\right),
$$

that is,

$$
[H(z(t))]^{\Delta} \leq \frac{z^{\Delta}(t)}{\omega\left(\left(\bar{G}^{-1}(z(t))\right)^{\frac{1}{p}}\right)} \leq g(t) .
$$

Integrating (64) from $t_{0}$ to $t$ yields

$$
H(z(t))-H\left(z\left(t_{0}\right)\right) \leq \int_{t_{0}}^{t} g(s) \Delta s .
$$

Since $H$ is increasing, and $z\left(t_{0}\right)=\bar{G}(C)+\int_{t_{0}}^{T} f(s) \Delta s$, then (65) implies

$$
z(t) \leq H^{-1}\left[H\left(\bar{G}(C)+\int_{t_{0}}^{T} f(s) \Delta s\right)+\int_{t_{0}}^{t} g(s) \Delta s\right], \quad t \in\left[t_{0}, T\right] \cap \mathbf{T} .
$$

Combining (57), (63), and (66), we obtain

$$
u(t) \leq\left\{\bar{G}^{-1}\left\{H^{-1}\left[H\left(\bar{G}(C)+\int_{t_{0}}^{T} f(s) \Delta s\right)+\int_{t_{0}}^{t} g(s) \Delta s\right]\right\}\right\}^{\frac{1}{p}}, \quad t \in\left[t_{0}, T\right] \cap \mathbf{T} .
$$

Taking $t=T$ in (67), and since $T$ is an arbitrary number in $\mathbf{T}_{0}$, then the desired inequality can be obtained after substituting $T$ with $t$. 
Remark 2.5: If we take $\mathbf{T}=\mathbf{R}, \tau_{1}(t)=\tau_{2}(t)$, then we can obtain a new bound of for the unknown continuous function $u(t)$, which is different from the result using the method in [[19], Theorem 2.1].

Remark 2.6: If we take $\mathbf{T}=\mathbf{R}$ in Theorem 2.3-2.4, or take $\mathbf{T}=\mathbf{Z}$ in Theorem 2.32.5 , then immediately we obtain a number of corollaries on continuous and discrete analysis, which are omitted here.

\section{Applications}

In this section, we will present some applications for the established results above. Some new bounds for solutions of certain dynamic equations on time scales will be derived in the following examples.

Example 1: Consider the delay dynamic integral equation on time scales

$$
u^{p}(t)=C+\int_{t_{0}}^{t} F(s, u(\tau(s))) \Delta s, \quad t \in \mathbf{T}_{0},
$$

with the initial condition

$$
\left\{\begin{array}{l}
u(t)=\phi(t), t \in\left[\alpha, t_{0}\right] \cap \mathbf{T}, \\
|\phi(\tau(t))| \leq|C|^{\frac{1}{p}}, \quad \forall t \in \mathbf{T}_{0}, \quad \tau(t) \leq t_{0},
\end{array}\right.
$$

where $u \in C_{r d}\left(\mathbf{T}_{0}, \mathbf{R}\right), C=u^{p}\left(t_{0}\right), p$ is a positive number with $p \geq 1, \tau, \alpha$ are defined as in Theorem 2.1, $\varphi \in C_{r d}\left(\left[\alpha, t_{0}\right] \cap \mathbf{T}, \mathbf{R}\right)$.

Theorem 3.1 Suppose, $u(t)$ is a solution of (68) and assumes $|F(t, u)| \leq f(t)|u|$, where $f \in C_{r d}\left(\mathbf{T}_{0}, \mathbf{R}_{+}\right)$, then we have

$$
|u(t)| \leq\left\{G^{-1}\left[G(|C|)+\int_{t_{0}}^{t} f(s) \Delta s\right]\right\}^{\frac{1}{p}}, \quad t \in \mathbf{T}_{0},
$$

where

$$
G(v)=\int_{1}^{v} \frac{1}{r^{\frac{1}{p}}} d r, \quad v>0
$$

Proof: From (68), we obtain

$$
|u(t)|^{p} \leq|C|+\int_{t_{0}}^{t}|F(s, u(\tau(s)))| \Delta s \leq|C|+\int_{t_{0}}^{t} f(s)|u(\tau(s))| \Delta s .
$$

Let $\omega \in C\left(\mathbf{R}_{+}, \mathbf{R}_{+}\right)$, and $\omega(v)=v$. Then, (72) can be rewritten as

$$
|u(t)|^{p} \leq|C|+\int_{t_{0}}^{t} f(s) \omega(|u(\tau(s))|) \Delta s .
$$

A suitable application of Theorem 2.1 to (73) yields the desired inequality.

Remark 3.1: In the proof for Theorem 3.1, if we apply Theorem 2.2 instead of Theorem 2.1 to (73), then we obtain another bound for $u(t)$ as follows.

$$
|u(t)| \leq\left\{|C|+\widetilde{G}^{-1}\left[\widetilde{G}(A(t))+\int_{t_{0}}^{t} f(s) \frac{1}{p} K^{\frac{1-p}{p}} \Delta s\right\}^{\frac{1}{p}}, t \in \mathbf{T}_{0},\right.
$$


where $K>0$ ia an arbitrary constant, and

$$
\left\{\begin{array}{l}
\widetilde{G}(v)=\int_{1}^{v} \frac{1}{r} d r, v>0, \\
A(t)=\int_{t_{0}}^{t} f(s)\left(\frac{1}{p} K^{\frac{1-p}{p}}|C|+\frac{p-1}{p} K^{\frac{1}{p}}\right) \Delta s .
\end{array}\right.
$$

Example 2: Consider the following delay dynamic differential equation on time scales

$$
\left(u^{p}(t)\right)^{\Delta}=F\left(t, u\left(\tau_{1}(t)\right), \int_{t_{0}}^{t} M\left(\xi, u\left(\tau_{2}(\xi)\right)\right) \Delta \xi\right), \quad t \in \mathbf{T}_{0},
$$

with the initial condition

$$
\left\{\begin{array}{l}
u(t)=\phi(t), t \in\left[\alpha, t_{0}\right] \cap \mathbf{T}, \\
\left|\phi\left(\tau_{i}(t)\right)\right| \leq|C|^{\frac{1}{p}}, \quad \forall t \in \mathbf{T}_{0}, \quad \tau_{i}(t) \leq t_{0}, \quad i=1,2,
\end{array}\right.
$$

where $u \in C_{r d}\left(\mathbf{T}_{0}, \mathbf{R}\right), C=u^{p}\left(t_{0}\right), p$ is a positive number with $p \geq 1, \alpha, \tau_{i}, i=1,2$ are defined as in Theorem 2.3, $\varphi \in C_{r d}\left(\left[\alpha, t_{0}\right] \cap \mathbf{T}, \mathbf{R}\right)$.

Theorem 3.2: Suppose $u(t)$ is a solution of (76), and assume $|F(t, u, v)| \leq f(t)|u|+|v|$, $|M(t, u)| \leq h(t)|u|$, where $f, h \in C_{r d}\left(\mathbf{T}_{0}, \mathbf{R}_{+}\right)$, then have

$$
|u(t)| \leq\left\{G^{-1}\left\{G(|C|)+\int_{t_{0}}^{t}\left[f(s)+\int_{t_{0}}^{s} h(\xi) \Delta \xi\right] \Delta s\right\}\right\}^{\frac{1}{p}}, \quad t \in \mathbf{T}_{0},
$$

where $G$ is defined as in Theorem 3.1.

Proof: The equivalent integral form of (75)-(76) can be denoted by

$$
u^{p}(t)=C+\int_{t_{0}}^{t} F\left(s, u\left(\tau_{1}(s)\right), \int_{t_{0}}^{s} M\left(\xi, u\left(\tau_{2}(\xi)\right)\right) \Delta \xi\right) \Delta s .
$$

Then,

$$
\begin{aligned}
|u(t)|^{p} & \leq|C|+\int_{t_{0}}^{t}\left|F\left(s, u\left(\tau_{1}(s)\right), \int_{t_{0}}^{s} M\left(\xi, u\left(\tau_{2}(\xi)\right)\right) \Delta \xi\right)\right| \Delta s \\
& \leq|C|+\int_{t_{0}}^{t}\left[f(s)\left|u\left(\tau_{1}(s)\right)\right|+\left|\int_{t_{0}}^{s} M\left(\xi, u\left(\tau_{2}(\xi)\right)\right) \Delta \xi\right|\right] \Delta s \\
& \leq|C|+\int_{t_{0}}^{t}\left[f(s)\left|u\left(\tau_{1}(s)\right)\right|+\int_{t_{0}}^{s}\left|M\left(\xi, u\left(\tau_{2}(\xi)\right)\right)\right| \Delta \xi\right] \Delta s \\
& \leq|C|+\int_{t_{0}}^{t}\left[f(s)\left|u\left(\tau_{1}(s)\right)\right|+\int_{t_{0}}^{s} h(\xi)\left|u\left(\tau_{2}(\xi)\right)\right| \Delta \xi\right] \Delta s \\
& =|C|+\int_{t_{0}}^{t}\left[f(s) \omega\left(\left|u\left(\tau_{1}(s)\right)\right|\right)+\int_{t_{0}}^{s} h(\xi) \omega\left(\left|u\left(\tau_{2}(\xi)\right)\right|\right) \Delta \xi\right] \Delta s,
\end{aligned}
$$

where $\omega \in C\left(\mathbf{R}_{+}, \mathbf{R}_{+}\right)$, and $\omega(u)=u$.

A suitable application of Theorem 2.3 to (79) yields the desired inequality.

\section{Conclusions}

In this paper, some new integral inequalities on time scales have been established. As one can see through the present examples, the established results are useful in dealing with the boundedness of solutions of certain delay dynamic equations on time scales. 
Finally, we note that the process of Theorem 2.1-2.5 can be applied to establish delay integral inequalities with two independent variables on time scales.

\section{Acknowledgements}

This work is supported by National Natural Science Foundation of China (11026047 and 10571110), Natural Science Foundation of Shandong Province (ZR2009AM011, ZR2010AQ026, and ZR2010AZ003) (China) and Specialized Research Fund for the Doctoral Program of Higher Education (20103705 110003)(China). The authors thank the referees very much for their careful comments and valuable suggestions on this paper.

\section{Author details}

${ }^{1}$ School of Mathematical Sciences, Qufu Normal University, Qufu, Shandong, 273165, China ${ }^{2}$ School of Science, Shandong University of Technology, Zibo, Shandong, 255049, China

\section{Authors' contributions}

QF carried out the main part of this article. All authors read and approved the final manuscript.

\section{Competing interests}

The authors declare that they have no competing interests.

Received: 16 February 2011 Accepted: 5 August 2011 Published: 5 August 2011

\section{References}

1. Hilger, S: Analysis on measure chains-a unified approach to continuous and discrete calculus. Results Math. 18, 18-56 (1990)

2. Bohner, M, Erbe, L, Peterson, A: Oscillation for nonlinear second order dynamic equations on a time scale. J Math Anal Appl. 301(2), 491-507 (2005). doi:10.1016/j.jmaa.2004.07.038

3. Xing, Y, Han, M, Zheng, G: Initial value problem for first-order integro-differential equation of Volterra type on time scales. Nonlinear Anal.: Theory Methods Appl. 60(3), 429-442 (2005)

4. Agarwal, RP, Bohner, M, O'Regan, D, Peterson, A: Dynamic equations on time scales: a survey. J Comput Appl Math $141(1-2), 1-26(2006)$

5. Wong, FH, Yeh, CC, Lian, WC: An extension of Jensen's inequality on time scales. Adv Dyn Syst Appl. 1(1), 113-120 (2006)

6. Cheng, XL: Improvement of some Ostrowski-Grüss type inequalities. Comput Math Appl. 42, 109-114 (2001). doi:10.1016/S0898-1221(01)00135-3

7. Bohner, M, Matthews, T: The Grüss inequality on time scales. Commun Math Anal. 3(1), 1-8 (2007)

8. Ngô, QA: Some mean value theorems for integrals on time scales. Appl Math Comput. 213, 322-328 (2009). doi:10.1016/j.amc.2009.03.025

9. Liu, WJ, Ngô, QA: Some lyengar-type inequalities on time scales for functions whose second derivatives are bounded. Appl Math Compu. 216, 3244-3251 (2010). doi:10.1016/j.amc.2010.04.049

10. Liu, WJ, Ngô, QA: A generalization of Ostrowski inequality on time scales for k points. Appl Math Comput. 203, 754-760 (2008). doi:10.1016/j.amc.2008.05.124

11. Li, WN: Some delay integral inequalities on time scales. Comput Math Appl. 59, 1929-1936 (2010). doi:10.1016/j. camwa.2009.11.006

12. $\mathrm{Ma}, \mathrm{QH}$, Pečarić, J: The bounds on the solutions of certain two-dimensional delay dynamic systems on time scales. Comput Math Appl. 61, 2158-2163 (2011). doi:10.1016/j.camwa.2010.09.001

13. Li, WN: Some new dynamic inequalities on time scales. J Math Anal Appl. 319, 802-814 (2006). doi:10.1016/j. jmaa.2005.06.065

14. Bohner, M, Peterson, A: Dynamic Equations on Time Scales: An Introduction with Applications. Birkhäuser, Boston (2001)

15. Jiang, FC, Meng, FW: Explicit bounds on some new nonlinear integral inequality with delay. J Comput Appl Math. 205, 479-486 (2007). doi:10.1016/j.cam.2006.05.038

16. Agarwal, R, Bohner, M, Peterson, A: Inequalities on time scales: a survey. Math Inequal Appl. 4(4), $535-557$ (2001)

17. Lipovan, O: Integral inequalities for retarded Volterra equations. J Math Anal Appl. 322, 349-358 (2006). doi:10.1016/j. jmaa.2005.08.097

18. Pachpatte, BG: On some new inequalities related to a certain inequality arising in the theory of differential equations. J Math Anal Appl. 251, 736-751 (2000). doi:10.1006/jmaa.2000.7044

19. Sun, YG: On retarded integral inequalities and their applications. J Math Anal Appl. 301, 265-275 (2005). doi:10.1016/j. jmaa.2004.07.020

doi:10.1186/1029-242X-2011-29

Cite this article as: Feng et al: Some nonlinear delay integral inequalities on time scales arising in the theory of

dynamics equations. Journal of Inequalities and Applications 2011 2011:29. 